\title{
Adolescents and smoking: The first puff may be the worst
}

\author{
Jonathan D. Klein
}

$\infty$

See related article page 255

$\mathrm{T}$ obacco use is the leading cause of preventable death globally, contributing to more than 5 million estimated deaths every year. ${ }^{1}$ Nicotine is a highly addictive substance, and tobacco remains the only legal product that, when used as intended, kills. Most smokers first use tobacco between the ages of II and I3, with I0\%-I5\% starting after age $19 .{ }^{2}$ Adolescence and young adulthood are a time of experimentation and transition in many behaviours, including smoking, which leads to opportunities for more effective prevention and cessation programs for youth.

In this issue, Gervais and colleagues ${ }^{3}$ report on the natural history and course of onset of cigarette use among grade 7 students. They describe addiction patterns and behavioural transitions in tobacco use using data from self-reported surveys conducted every 3 months over a 5 -year study period. Contrary to older, common assumptions that experimentation leads to regular use and then to addiction, the authors found that symptoms of nicotine dependence develop early in the course of an adolescent's exposure to cigarettes. In fact, for some of the participants, signs of addiction developed quickly, even after a single first puff, and most youth reported symptoms of addiction and cravings long before becoming a daily smoker.

In the past decade, some public health advocates have developed a false sense of security toward tobacco. The move to implement legislation for clean indoor and outdoor air has progressed successfully in many locations in North America and elsewhere in the world. This trend reflects the general social consensus that tobacco smoke is irritating, growing awareness that secondhand tobacco smoke is harmful and expectations that these exposures can and should be prevented. However, college-aged young adults (aged I8-24) continue to be targeted by tobacco companies in North America, children continue to be influenced by pro-smoking imagery in television and film media, and tobacco promotion influences children to initiate tobacco use. ${ }^{4}$ The most recently released data from the US Centers for Disease Control and Prevention show that, in 2005, 23\% of high school students reported smoking in the past month. That year marked the first year without a decline in the rate of smoking among youth since the rate peaked at $36 \%$ in $1997 .{ }^{2}$ Clearly, we cannot afford to remain lax about this health hazard.

The study by Gervais and colleagues provides important independent confirmation that youth are at special risk of nicotine addiction. Their findings confirm those from earlier work ${ }^{5}$ and should heighten our awareness of the dangerous addictive potential of this substance. Early onset of addiction is not really a surprise; however, the fact that the old "it's okay to experiment" message is not based on evidence and that experimentation has a high potential for harm need to be emphasized in public health, education and clinical settings for children and their families.

There is strong evidence that adult smokers can successfully quit and that counselling and pharmacotherapy help them succeed. Counselling, nicotine replacement and buproprion increase the chances for success, especially when used together. Meta-analyses of randomized controlled trials involving adult smokers have shown increasing rates of cessation in response to increasing counselling intensity and time. ${ }^{6}$ Counselling and behavioural therapies associated with higher rates of cessation include problem solving and skills training, provision of social support as part of treatment, and help obtaining social support outside of treatment. Self-help interventions, telephone counselling, tailored materials, and combining nicotine replacement with other interventions have also been shown to be effective. ${ }^{6} \mathrm{Al}-$ though the evidence base for cessation counselling for adolescents remains relatively weak, the data reported by Gervais and colleagues provide fresh, important evidence that the best course of action is for youth never to start smoking. In addition, youth are known to be sensitive to interventions that affect access to cigarettes, such as price increases (including deliberate increases in taxation) and enforcement of age bans on sales to youth. ${ }^{7}$

Nearly $75 \%$ of youth smokers have seriously thought about quitting, $56 \%$ of high school and $50 \%$ of college smokers have tried to quit in the past year, and most of those who have tried to quit have failed. ${ }^{7}$ Most adolescent smokers know they are addicted and want to quit. However, young people do not think there are resources to help them quit, many clinicians do not feel well prepared to counsel adolescents about quitting smoking, and resources for adolescent cessation have are not widely available. ${ }^{7}$

This new evidence should strengthen the resolve of public health authorities and local governments to prevent children and youth from starting to smoke in the first place and to protect them against exposure to smoking, either in the form of secondhand smoke or pro-smoking imagery in the media. 


\section{This article has been peer reviewed.}

Jonathan Klein is Director of the American Academy of Pediatrics Julius B. Richmond Center of Excellence for Children and Secondhand Smoke and is Associate Professor of Pediatrics and of Community and Preventive Medicine, University of Rochester School of Medicine, Rochester, NY.

Competing interests: None declared.

Acknowledgement: Jonathan Klein's work on this commentary was supported in part by the Flight Attendant Medical Research Institute and by the American Legacy Foundation.

\section{REFERENCES}

I. World Health Organization. Statement from Catherine Le Galès - Camus, Assistant Director-General, Non Communicable Diseases and Mental Health. Available: www .who.int/tobacco/communications/events/wntd/2006/statement/en/index.html (accessed 2006 Jun 27).

2. US Centers for Disease Control and Prevention. Trends in the prevalence of cigarette use. Available: www.cdc.gov/HealthyYouth/yrbs/pdf/trends/2005_YRBS
Cigarette_Use.pdf (accessed 2006 Jun 27).

3. Gervais A, O'Loughlin J, Meshefedjian G, et al. Milestones in the natural course of onset of cigarette use among adolescents. CMAJ 2006;175(3):255-6I.

4. DiFranza JR, Wellman RJ, Sargent JD, et al; Tobacco Consortium, Center for Child Health Research of the American Academy of Pediatrics. Tobacco promotion and the initiation of tobacco use: assessing the evidence for causality. Pediatrics 2006; II7:eI237-48.

5. DiFranza JR, Savageau JA, Fletcher K, et al. Measuring the loss of autonomy over nicotine use in adolescents: the DANDY (Development and Assessment of Nicotine Dependence in Youths) study. Arch Pediatr Adolesc Med 2002;156:397-403.

6. Fiore MC, Bailey WC, Cohen SJ, et al. Treating tobacco use and dependence. Clinical Practice Guideline. Rockville (MD): Public Health Service, US Department of Health and Human Services; June 2000. Available: www.surgeongeneral.gov /tobacco/treating tobacco_use.pdf (accessed 2006 June 29).

7. Pbert L, Moolchan ET, Muramoto M, et al; Tobacco Consortium, Center for Child Health Research of the American Academy of Pediatrics. The state of office-based interventions for youth tobacco use. Pediatrics 2003;inI:e650-6o.

Correspondence to: Dr. Jonathan D. Klein, University of Rochester School of Medicine, 69o-6or Elmwood Ave., Rochester NY I4642, USA; fax 585 242-9733; jonathan_klein@urmc.rochester.edu

\title{
Personal protective equipment for preventing respiratory infections: What have we really learned?
}

\author{
John M. Conly
}

$\infty$

See related article page 249

$\mathrm{T}$ he use of personal protective equipment (PPE) for health care workers (HCWs) has evolved from the isolation precautions first implemented years ago for patients with communicable diseases such as smallpox, tuberculosis and diphtheria. ${ }^{1}$ The use of PPE (gloves, gowns, masks and eye protection) in combination with single rooms with airflow control represents the usual barrier precautions employed to prevent transmission of pathogenic mircoorganisms to HCWs. The mechanisms of transmission (airborne, droplet, contact, vector or common vehicle) for the microbe in question often mandate the specific combination of barrier precautions chosen. $^{2}$

Reports of SARS among HCWs in hospital outbreaks reported from Canada, China, Hong Kong, Taiwan and Vietnam focused attention on the critical importance of infectioncontrol practices, including the use of PPE, and the role of training and knowledge among HCWs in using PPE and barrier precautions appropriately. ${ }^{3}$ Microbes transmitted by the airborne or droplet routes create the greatest anxiety among HCWs. Additional risks for transmission are posed by $n$ the emergence of new pathogens with a severe illness profile (e.g., SARS and avian influenza) and immuno- and other highly compromised patients, who may carry greater microbial burdens for prolonged periods. The advent of new technological diagnostic and therapeutic modalities may also lengthen HCWs' exposure to patients carrying highly infective pathogens.
A thorough understanding of the usual routes of transmission of microbes and the conditions under which these routes may change is paramount to prevent the spread of an infection. ${ }^{2}$ Contact transmission, the most common route, occurs when microbes are transferred either directly by physical contact between an infected or colonized individual and a new host or indirectly via an intermediate object (a fomite). ${ }^{2}$ Droplet transmission involves drops of fluid $5 \mu \mathrm{m}$

\section{SARS focused attention on}

\section{the critical importance of infection-control practices.}

in diameter and larger, produced from the respiratory tract during coughing or sneezing or by medical procedures, propelled within $1 \mathrm{~m}$ of the source patient. Airborne transmission refers to dissemination of microbes within droplet nuclei (particles $<5 \mu \mathrm{m}$ in diameter), which result from the evaporation of larger droplets or exist within dust particles and remain suspended in the air for long periods. Although most respiratory viruses are transmitted by droplet and contact methods, microbes that can spread via airborne trans- 